\title{
Molecular and Histologic Evidence of Novel Erectile Dysfunction Rat Model as an Aging Atherosclerosis Model: A Preliminary Study
}

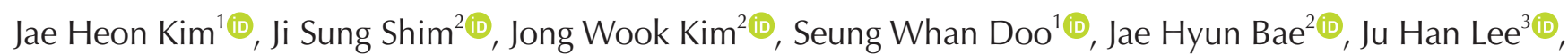
Yun Seob Song ${ }^{1}$, Je Jong $\mathrm{Kim}^{2}$ (i) , Du Geon Moon ${ }^{2}$ (iD

${ }^{1}$ Department of Urology, Soonchunhyang University Hospital, Soonchunhyang University College of Medicine, ${ }^{2}$ Department of Urology, Korea University College of Medicine, Seoul, ${ }^{3}$ Department of Pathology, Korea University Ansan Hospital, Korea University College of Medicine, Ansan, Korea

Purpose: To validate a novel arteriogenic erectile dysfunction (ED) model with atherosclerosis (AS) based on molecular and histologic evidence induced by chronic pelvic ischemia (CPI) and determine effect of phosphodiesterase-5 inhibitor treatment.

Materials and Methods: Twenty 16-week-old male Sprague-Dawley rats were divided into three experimental groups (Group I, untreated sham-operated rats with regular diet; Group II, CPI with cholesterol diet; Group III, CPI model with cholesterol diet and mirodenafil). Erectile function was accessed using maximum intracavernous pressure (ICP) and ICP/mean arterial pressure (MAP). Molecular changes were examined by western blot analysis using hypoxia inducible factor 1-alpha (HIF-1 $\alpha)$, endothelial nitric oxide synthase (eNOS), and transforming growth factor beta-1 (TGF- $\beta 1$ ) antibodies. Collagen change was evaluated by Masson's trichrome staining.

Results: In vivo measurements of ICP and ICP/MAP in Group II were significantly lower than those in Group I $(p<0.01)$. Smooth muscle/collagen ratio in Group II was significantly lower than that in Group I $(p<0.05)$. After treatment with mirodenafil for four weeks, Group III showed significantly higher levels of ICP and ICP/MAP than Group II ( $<<0.05)$. Western blot analysis showed that HIF-1 $\alpha$ and TGF- $\beta 1$ levels were significantly higher in Group II whereas eNOS levels were significantly lower in Group II than those in Group I or III.

Conclusions: A novel arteriogenic ED with AS model is successfully induced by CPI and validated based on molecular and histologic evidences.

Keywords: Atherosclerosis; Erectile dysfunction; Phosphodiesterase 5 Inhibitors; Vasculogenic impotence

This is an Open Access article distributed under the terms of the Creative Commons Attribution Non-Commercial License (http://creativecommons.org/licenses/by-nc/4.0) which permits unrestricted non-commercial use, distribution, and reproduction in any medium, provided the original work is properly cited.

\section{INTRODUCTION}

Among etiologies of erectile dysfunction (ED), arteriogenic $\mathrm{ED}$ is the most common etiology. Considering that increasing population of aging groups have ED, atherosclerosis (AS) originated arteriogenic ED could be the most popular etiology among older groups. Treating ED could be a medical burden [1,2].

Received: Mar 12, 2019 Revised: Apr 28, 2019 Accepted: May 7, 2019 Published online Jun 14, 2019

Correspondence to: Du Geon Moon iD https://orcid.org/0000-0002-9031-9845

Department of Urology, Korea University Guro Hospital, Korea University College of Medicine, 148 Gurodong-ro, Guro-gu, Seoul, 08308 Korea.

Tel: +82-2-2626-3201, Fax: +82-2-2626-1321, E-mail: dgmoon@korea.ac.kr 
Considering the pivotal role of preclinical studies in investigating the fundamental mechanism of arteriogenic $\mathrm{ED}$ with $\mathrm{AS}$, an in vivo model that is broadly validated is essential for further research. Currently, there is limited report on such effort. Moreover, there exist difficulties regarding feasibility and reproducibility of such model in the literature [3-10]. Furthermore, reported models have limitations including no endothelial dysfunction unless genetic modification or a special diet is required to evoke endothelial injury. Inducing mechanical injury is feasible by ligation or clamping of iliac arteries [3-5]. However, to induce endothelial dysfunction is not easy. Moreover, ligation or clamping is far from physiologic AS which reflects progressive luminal narrowing.

To overcome limitations of previous studies validating an aging arteriogenic ED model, we adopted chronic pelvic ischemia (CPI). Our previous experience has shown that such CPI model is a sound in vivo model of an aging ischemic bladder [11-14]. In addition, we have examined an arteriogenic ED model with $\mathrm{AS}$ in a pilot preclinical study [15].

The aim of the present study was to validate our novel arteriogenic ED model with AS based on molecular and histologic evidence and determine the effect of treatment with a phosphodiesterase-5 (PDE-5) inhibitor.

\section{MATERIALS AND METHODS}

\section{Animal subjects}

Male Sprague-Dawley rats (400-450 g) at 16 weeks of age were obtained, randomized, and acclimated for two weeks in plastic cages (two rats per cage). Rats were provided free access to purified water and standard compressed feed. Room air temperature was maintained at $22^{\circ} \mathrm{C} \pm 1^{\circ} \mathrm{C}$ and light was provided from 7 a.m. to 7 p.m.

\section{Ethics statement}

This study was approved by the Institutional Animal Care and Use Committee of Korea University (No. KUIACUC-20130523-3). Animal handling followed animal experimentation guide provided by the Animal Laboratory of Korea University, Ansan Hospital.

\section{Experimental protocols}

There were three experimental groups: untreated sham-operated rats with a regular diet (Group I, control [n=7]), CPI with cholesterol diet (Group II, ischemia [n=6]), and CPI model with cholesterol diet and mirodenafil treatment (Group III, ischemia with treatment [n=7]). Rats of Groups II and III received an endothelial injury of iliac arteries and $2 \%$ cholesterol diet for eight weeks. Group I rats underwent a sham operation. They were maintained with a regular diet. Groups II and III rats received an endothelial injury with cholesterol diet during the eighth week. Eight weeks after operation, Group III rats were fed with mirodenafil plus normal saline for four weeks. Group II rats were fed with normal saline only. Twelve weeks after operation, in vivo measurements of parameters associated with erectile function (maximum intracavernous pressure $[\mathrm{ICP}]$ and ICP/mean arterial pressure [MAP]) were performed. Mirodenafil (5-Ethyl-3,5-dihydro-2-[5-([4-(2hydroxyethyl)-1-piperazinyl]sulfonyl)-2-propoxyphenyl]7-propyl-4H-pyrrolo[3,2-d]pyrimidin-4-one) was provided by SK Chemical Ltd. (Seoul, Korea) with a purity of $99 \%$. We dissolved mirodenafil using $40 \%$ polyethylene glycol-electrolyte solution in water and used it at a dose of $20 \mathrm{mg} / \mathrm{kg}$. The solution $(0.3-0.5 \mathrm{~mL})$ was administered via an oral Zonde needle.

\section{In vivo chronic pelvic ischemia model}

An in vivo CPI model followed the original model introduced by Nomiya et al [11-14]. After general anesthesia using isoflurane (1.5\%-2.5\%), an inguinal incision was made. Exposure of the iliac fossa and femoral artery was made and a 2-Fr Fogarty arterial embolectomy catheter (E-060-2F; Edwards Lifesciences LLC, Irvine, CA, USA) was inserted through a small incision in the femoral artery. After upward placement of the catheter to the fossa area, air inflation $(0.2 \mathrm{~mL})$ was done and pulled downward to the initiation location of the femoral artery. This manual pulling down was repeated 10 times to induce mechanical intimal injury. The same approach was performed on the contralateral region. For the control group, a sham operation was done in the same manner without inducing mechanical intimal injury.

\section{In vivo measurement of intracavernous pressure}

In vivo measurement of ICP was performed under general anesthesia with isoflurane $(1.5 \%-2.5 \%)$ at 12 weeks after establishment of CPI or sham CPI opera- 
tion. To measure MAP, a 24-gauge angiocatheter was inserted into the left carotid artery. After exposure of the penis corpus cavernosum, an incision was extended to the low abdominal area to introduce the bladder and prostate. A 22-gauge needle was inserted into the corpus cavernosum artery and connected to a polyethylene tube (PE 50; Clay Adams, Parsippany, NJ, USA). After visual confirmation of the right major pelvic ganglion, a bipolar electrical stimulator (Grass SD9;
Grass Instrument, Quincy, MA, USA) was placed and the cavernosal nerve was stimulated for 50 seconds with the condition of $5 \mathrm{v}, 20 \mathrm{~Hz}$, and 0.5 milliseconds. Two cycles of electrical stimulation were done and the interval of each stimulation was maintained for at least 5 minutes. Main parameters included maximal ICP, ICP/MAP, total ICP, and slope. Measurements were done using AD Instrument PowerLab ${ }^{\mathrm{TM}}$ (AD Instrument, Colorado Springs, CO, USA).
A

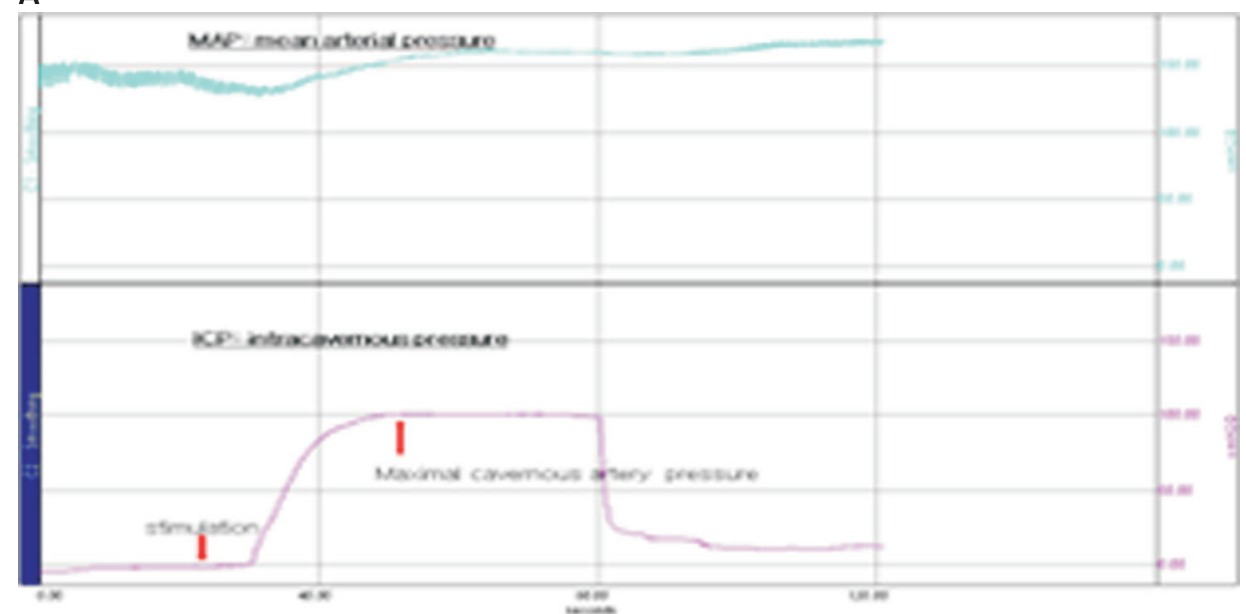

B

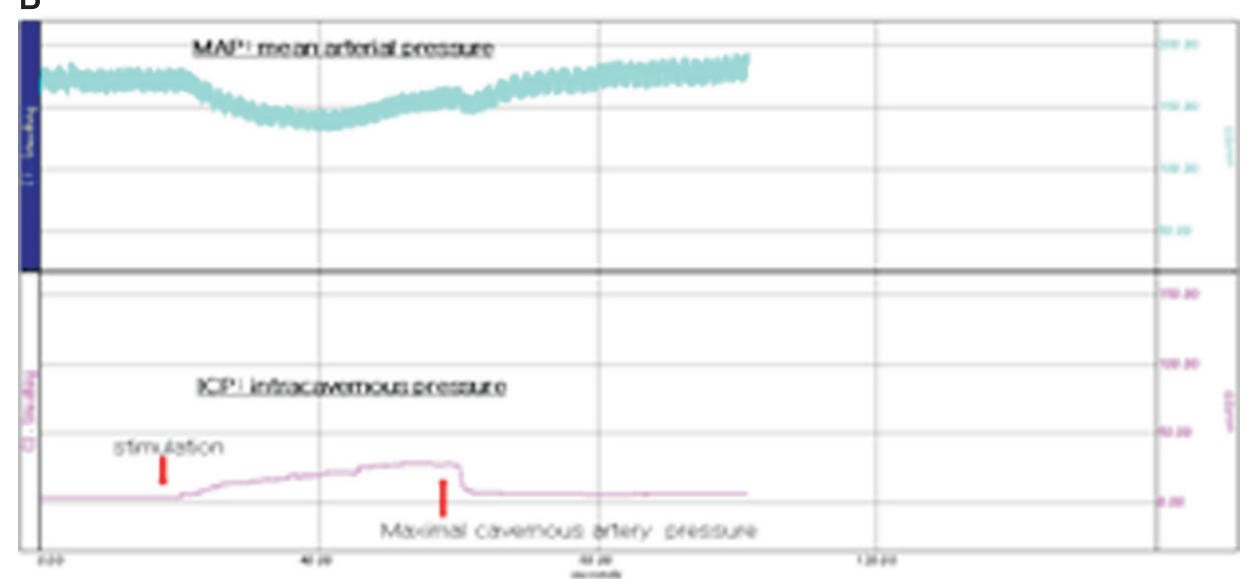

C

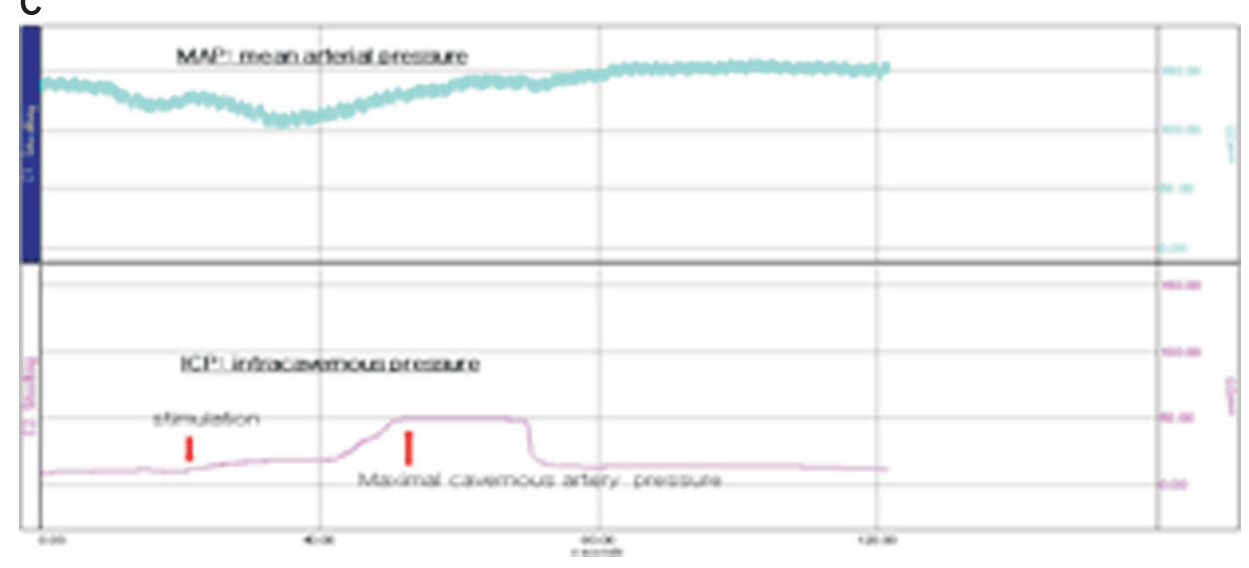

Fig. 1. Representative charts of intracavernosal pressure (ICP). Increase in ICP by electrical stimulation was observed in the sham group $(n=7 ; A)$. Increases in ICP were clearly lower in the chronic pelvic ischemia (CPI) model group $(n=6 ; B)$. Increases in ICP were higher than the CPI but lower than the sham group in $\mathrm{CPI}$ with treated group $(n=7, C)$. The upper curve is mean arterial pressure and the lower curve is ICP. 


\section{Western blot analysis}

Sample preparation was done by homogenizing sample tissues that were kept under $-70^{\circ} \mathrm{C}$ and centrifuged at 12,000 rpm for 10 minutes. Protein concentrations were determined by Bradford solution (Sigma, St. Louis, MO, USA). The amount of each protein sample loaded was $40 \mu \mathrm{g}$. Sodium dodecyl sulfate (SDS) polyacrylamide gel electrophoresis was done for measuring levels of hypoxia inducible factor 1-alpha (HIF-1 $\alpha$ ), endothelial nitric oxide synthase (eNOS), and transforming growth factor beta-1 (TGF- $\beta 1$ ) by western blotting. For HIF-1 $\alpha$ and eNOS, 6\% SDS acrylamide gel was used with sampling loading amount of $40 \mu \mathrm{g}$. For settings of stacking gel and separation gel, $-80 \mathrm{~V}$ and $-100 \mathrm{~V}$ were adopted, respectively. For TGF- $\beta 1,12 \%$ SDS acrylamide gel was used with sampling loading amount of 40 $\mu \mathrm{g}$. For settings of stacking gel and separation gel, $-80 \mathrm{~V}$ and $-120 \mathrm{~V}$ were adopted, respectively. Transfer was performed using a polyvinylidene difluoride membrane at $150 \mathrm{~mA}$ for 90 minutes. Immunoblotting was performed after blocking and incubation with primary antibodies and secondary antibodies. Primary antibodies specific for HIF-1 $\alpha$, eNOS, and TGF- $\beta 1$ (Santa Cruz Biotechnology, Santa Cruz, CA, USA) at a dilution of 1:200 in Tris-buffered saline, 0.1\% Tween 20 buffer were incubated with the membrane overnight. Actin antibody (Cell Signaling, Danvers, MA, USA) was used at a dilution of 1:3,000 in $2.5 \%$ skim milk. It was incubated with the membrane at room temperature for 1 hour. For secondary antibodies, anti-rabbit (Sigma) was used at a dilution of 1:30,000. Finally, developing was done with an enhanced chemiluminescence solution.

\section{Vascular histology}

After obtaining a vascular specimen including ab- dominal aorta and iliac fossa, histological analysis was done. After fixation with a phosphate-buffered $10 \%$ formaldehyde solution, the specimen was used to prepare paraffin block. After preparing paraffin block sections (5 $\mu \mathrm{m}$ in thickness), H\&E staining was done. Intimal injury and lumen were then inspected.

\section{Masson trichrome staining}

After washing fixed fragment of corpus carvernosum, dehydration was done using serial ethanol solution. A paraffin block was made and sectioned to slices (10 $\mu \mathrm{m}$ in thickness). Slice was then placed on a gelatincoated glass for Masson trichrome staining. Quantitative analysis was done using an ECLIPSE Ti-U imaging analysis (Nikon, Tokyo, Japan) and NIS Elements D30 (Nikon). Calculation of smooth muscle (SM)/collagen ratio was done by repeating measurements 10 to 15 times under magnifying powers of 40 or 200 .

\section{Statistical analysis}

All data are described as mean \pm standard deviation. For analytic method, one-way ANOVA test was used. To simulate non-parametric test, a Kruskal-Wallis test was conducted. Differences were considered significant at $\mathrm{p}<0.05$. All analyses were performed using STATA ver. 14 (Stata Corp. LP, College Station, TX, USA).

\section{RESULTS}

\section{Intracavernous pressure}

Although the slope of each group showed various patterns (Fig. 1), maximal ICP showed prominent differences among groups I, II, and III (at 73.92 \pm 22.12 $\mathrm{mmHg}, 27.50 \pm 11.80 \mathrm{mmHg}$, and $39.68 \pm 7.92 \mathrm{mmHg}$, respectively, $\mathrm{p}=0.001$ ) (Table 1 ). ICP/MAP showed

Table 1. Comparative analysis of parameters from ICP measurements

\begin{tabular}{llccccc}
\hline \multicolumn{1}{c}{ Group } & Category & Maximal ICP & ICP/MAP & Total ICP & Slope & Total ICP/MAP \\
\hline Control & Mean \pm SD & $73.9214^{\mathrm{a}, \mathrm{c}} \pm 22.1212$ & $0.5223^{\mathrm{a}, \mathrm{c}} \pm 0.2158$ & $3,542.0000^{\mathrm{a}, \mathrm{c}} \pm 1,391.2937$ & $3.1000 \pm 2.4674$ & $24.3700^{\mathrm{a}, \mathrm{c}} \pm 10.0201$ \\
& Median & $77.3000^{\mathrm{a}, \mathrm{c}}$ & $0.4497^{\mathrm{a}, \mathrm{c}}$ & $3,266.0000^{\mathrm{a}, \mathrm{c}}$ & 2.3100 & $18.9900^{\mathrm{a}, \mathrm{c}}$ \\
\multirow{3}{*}{ Ischemia } & Mean $\pm \mathrm{SD}$ & $27.5033^{\mathrm{b}, \mathrm{c}} \pm 11.8015$ & $0.1590^{\mathrm{b}, \mathrm{c}} \pm 0.5667$ & $1,857.7833^{\mathrm{c}} \pm 910.3737$ & $1.1360 \pm 1.0683$ & $7.8602^{\mathrm{c}} \pm 1.9001$ \\
& Median & $29.5100^{\mathrm{b}, \mathrm{c}}$ & $0.1427^{\mathrm{b}, \mathrm{c}}$ & $1,829.5000^{\mathrm{c}}$ & 0.6070 & $7.3921^{\mathrm{c}}$ \\
Ischemia with & Mean $\pm S D$ & $39.6871^{\mathrm{a}, \mathrm{b}} \pm 7.9249$ & $0.2815^{\mathrm{a}, \mathrm{b}} \pm 0.1425$ & $1,512.5714^{\mathrm{a}} \pm 376.6394$ & $0.9768 \pm 0.7373$ & $11.9715^{\mathrm{a}} \pm 3.3952$ \\
treatment & Median & $38.4700^{\mathrm{a}, \mathrm{b}}$ & $0.2512^{\mathrm{a}, \mathrm{b}}$ & $1,555.0000^{\mathrm{a}}$ & 0.7120 & $11.7292^{\mathrm{a}}$ \\
p-value & & 0.001 & 0.002 & 0.006 & 0.059 & 0.020 \\
\hline
\end{tabular}

ICP: intracavernous pressure, MAP: mean arterial pressure, SD: standard deviation.

${ }^{a}$ Statistically significant between control group and ischemia with treatment group; ${ }^{b}$ statistically significant between ischemia group and ischemia with treatment group; 'statistically significant between control group and ischemia group. 
prominent differences among groups I, II, and III (at $0.5223 \pm 0.2158,0.1590 \pm 0.5667$, and $0.2815 \pm 0.1425$, respectively, $\mathrm{p}=0.002$ ). In post hoc analyses, the maximal ICP, ICP/MAP, total ICP, and total ICP/MAP of group I were higher than those of group II or group III (all $\mathrm{p}<0.05$ ). Between groups II and III, only maximal ICP

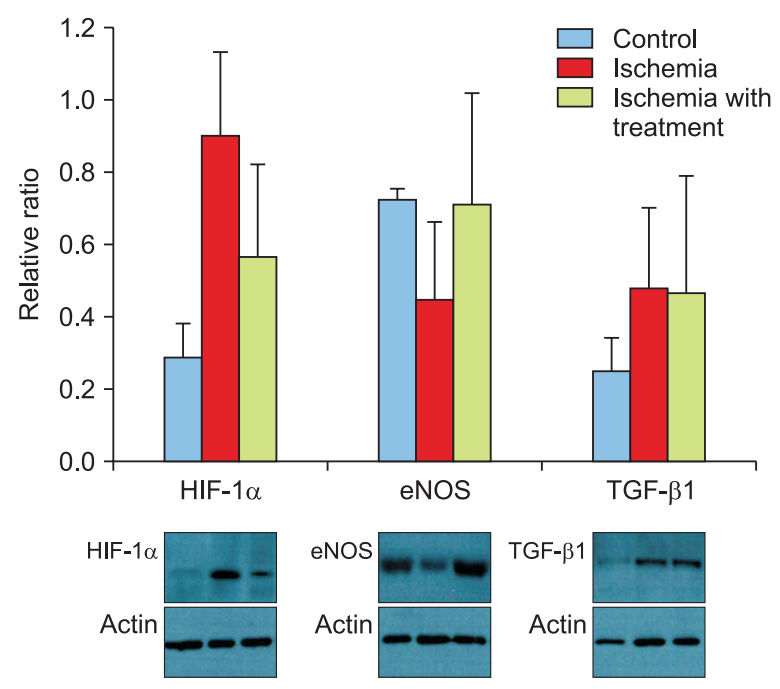

Fig. 2. Expression levels of molecular proteins in corpus cavernosum based on Western blotting. Alpha-smooth muscle actin ( $\alpha-S M A)$ was used as a control. HIF-1 $\alpha$ : hypoxia induced factor 1-alpha, eNOS: endothelial nitric oxide synthase, TGF- $\beta 1$ : transforming growth factor beta-1. and ICP/MAP showed significant differences $(p<0.05)$.

\section{Western blotting analysis}

Densitometry was used to obtain quantitative results of Western blot. Results were normalized to levels of actin (Fig. 2). HIF-1 $\alpha$ levels of group II were significantly higher than those of group I or group III $(0.9051 \pm 0.2286$ vs. $0.2933 \pm 0.0942$ or $0.5675 \pm 0.2586$, $\mathrm{p}=0.02$ ). eNOS levels of group II were significantly lower than those of group I or group III $(0.4527 \pm 0.2119$ vs. $0.7247 \pm 0.0339$ or $0.7144 \pm 0.3097, \mathrm{p}=0.04)$. TGF- $\beta 1$ levels of group I were significantly lower than those of group II or group III $(0.2547 \pm 0.0925$ vs. $0.4807 \pm 0.2284$ or $0.4718 \pm 0.3212, \mathrm{p}=0.03)$. There was no significant difference in TGF- $\beta 1$ level between groups II and III.

\section{Vascular histology}

A longitudinal section of common iliac arteries $(\times 400)$ revealed remarked luminal narrowing with intimal thickening and a degenerative change of media in the ischemia group compared to other groups (Fig. 3).

\section{Masson's trichrome staining}

The SM/collagen ratio of group I was significantly higher than that of group II or III $(0.182 \pm 0.05 v s$.
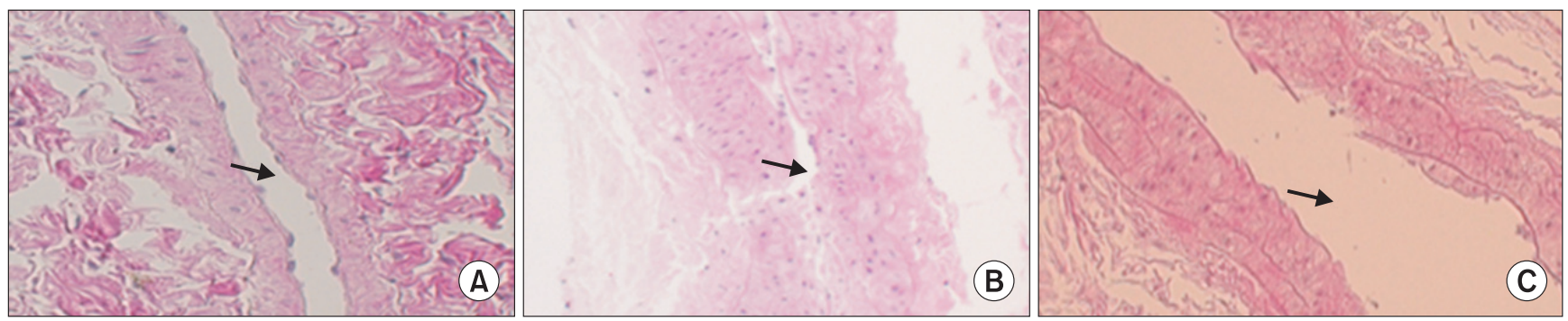

Fig. 3. Longitudinal section of common iliac arteries $(H \& E, \times 400)$. Luminal narrowing is prominent with intimal thickening in the ischemia group ( $B$ ) compared to that in the control group $(A)$ and ischemia with treatment group $(C)$. Arrows indicate arterial lumen.
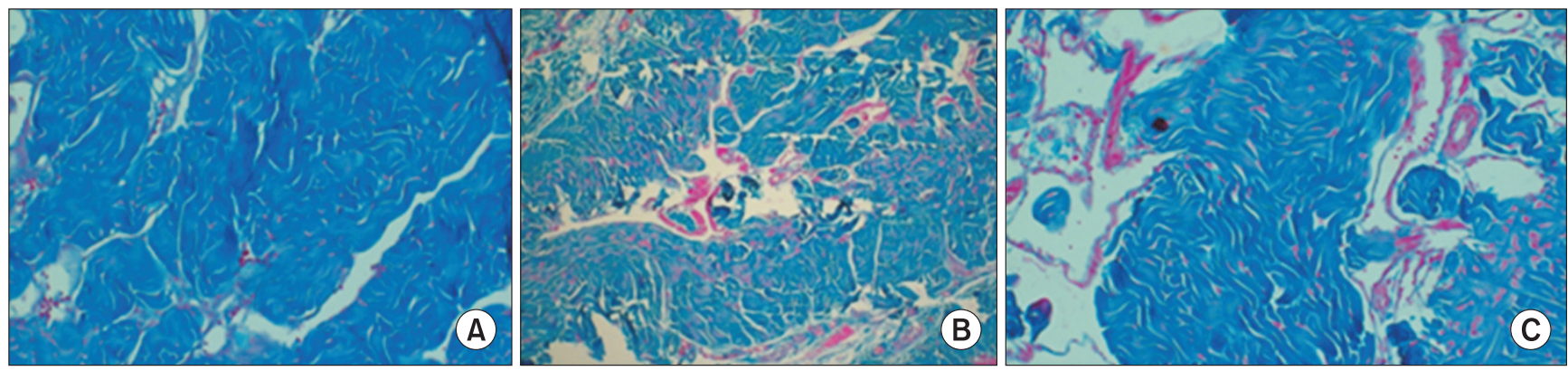

Fig. 4. Masson trichrome staining of penile corpus cavernosum $(\times 200)$. Smooth muscle/collagen ratio of the ischemia group (B) remained significantly lower than that in the control (A). The ischemia with treatment group (C) showed a higher ratio than that in the chronic pelvic ischemia group (middle). 
$0.0231 \pm 0.1918$ or $0.0441 \pm 0.0712$ ) (Fig. 4). However, there was no significant difference in $\mathrm{SM} /$ collagen ratio between groups II and III.

\section{DISCUSSION}

There is no doubt that AS is a significant etiology for ED $[1,2,16]$. It could be a grave issue for both individuals and public health considering its deep relationship with the aging process. Establishing a validated arteriogenic ED model with $\mathrm{AS}$ is the first step to clearly determine the mechanism and potential treatment for arteriogenic ED with AS. Based on our previous pioneer approach on this issue [15], this study demonstrated its validity with molecular and histological evidences, like the first time. To overcome limitations of previous studies on this issue, reviews were done to systematically design this validation study.

The key pathophysiology of AS change lies in obstructing arterial inflow by narrowed lumen due to artherosclerotic lesions $[1,4,10,17,18]$. Hence, without intimal injury, an ED model could not show endothelial dysfunction. The main mechanism of arteriogenic ED with AS could be explained with two components: structural and functional changes. Structural change is induced by impaired arterial flow which results in hypoxia and fibrosis. In this change, TGF- $\beta$ plays an important role [4,5]. Among previous studies, arterial ligation or partial obstruction was a good model to show structural change $[4,5,10]$. However, these studies did not try to show functional change associated with endothelial dysfunction. The key mechanism of endothelial dysfunction lies on NOS. Nitric oxide (NO) is involved in anti-inflammation, anti-platelet aggregation, and anti-oxidative function [8,9,18]. NOS is composed of three isoforms including neuronal NOS (nNOS), eNOS, and inducible NOS. eNOS is in the endothelium of the cavernosal artery. It has a critical role in the endothelial dysfunction mechanism.

Nowadays, these two main components (structural and functional changes) have a tendency to be overlapped [3,18]. Hence, validation of a promising ED model should include these two components together. Our model meets this demand well. Structural change was mediated by the progressive narrowing of the arterial lumen which is far more physiologic than previous artificial ligations or obstructions. To induce functional change, our model included a long-term cholesterol diet and progressive narrowing of the arterial lumen by intimal injury. This study had additional benefit by showing functional change. By in vivo measurement of ICP, western blotting of molecular markers, and histological analysis, our model was validated as a promising arteriogenic ED model with AS. Moreover, we used male rats at 16 weeks old in the randomization period, meaning that our model could reflect an aging model more properly than other studies.

Another feature of our study was that mirodenafil, a PDE-5 inhibitor, was used as an inner validation tool. Based on its expected effect on ICP, we could conclude that this model was properly established. By integrating all our results, we could conclude that impaired arterial flow was generated and it created secondary cavernosal dysfunction which was manifested by abnormal ICP and increased HIF-1 $\alpha$. This structural change was aggravated, resulting in fibrosis which also affected secondary cavernosal dysfunction and manifested by elevation of TGF- $\beta$ and decreased SM/collagen ratio. Together with structural change, there was also a functional change in that eNOS was decreased by endothelial dysfunction. One notable finding of our study was that structural change could not be reversed by PDE-5 inhibitor treatment.

Although this study validated a novel ED model, it had several limitations. First, there was no information about the critical role of mRNA, meaning that detailed processes could not be explained. For example, this study could not assert that endothelial dysfunction was preceded by fibrotic change. Second, besides eNOS, there are more molecular markers that can explain functional change, such as nNOS, nerve growth factor, and vascular endothelial growth factor [3]. Third, considering cholesterol resistant features of rat species, other efforts including genetic and longer-term cholesterol diet are needed. Lastly, our control group could not fully show whether characteristics of this model are caused by mechanical injury or cholesterol diet. Hence, additional groups (i.e., a group with real operation plus regular diet and a group with sham operation plus cholesterol diet) are needed to define characteristics of this model in the future.

\section{CONCLUSIONS}

This study validated a novel arteriogenic ED model with AS which was successfully induced by CPI based 
on molecular and histologic evidences. More promising preclinical studies using this model are needed to elucidate the fundamental pathophysiology and treatment for arteriogenic ED with AS.

\section{ACKNOWLEDGEMENTS}

This research was supported by Soonchunhyang University Fund (No. 20190005).

\section{Conflict of Interest}

The authors have nothing to disclose.

\section{Author Contribution}

Conceptualization: JHK, DGM. Funding acquisition: JHK. Methodology: JSS, JWK, SWD. Supervision: DGM, JHB, JHL, YSS, JJK. Writing-original draft: JHK, DGM. Writing-review \& editing: all authors.

\section{Data Sharing Statement}

The data analyzed for this study have been deposited in HARVARD Dataverse and are available at https://doi. org/10.7910/DVN/HBJCKF.

\section{REFERENCES}

1. Aboseif SR, Breza J, Orvis BR, Lue TF, Tanagho EA. Erectile response to acute and chronic occlusion of the internal pudendal and penile arteries. J Urol 1989;141:398-402.

2. Gandaglia G, Briganti A, Jackson G, Kloner RA, Montorsi F, Montorsi P, et al. A systematic review of the association between erectile dysfunction and cardiovascular disease. Eur Urol 2014;65:968-78.

3. De Young L, Bella A, Howard J, Brock G. Arteriogenic erectile dysfunction alters protein expression within the cavernosal tissue in an animal model. J Sex Med 2005;2:199-206.

4. Hotta Y, Hattori M, Kataoka T, Ohno R, Mikumo M, Maeda Y, et al. Chronic vardenafil treatment improves erectile function via structural maintenance of penile corpora cavernosa in rats with acute arteriogenic erectile dysfunction. J Sex Med 2011; 8:705-11.

5. Hotta Y, Ohno R, Kataoka T, Mikumo M, Takahata Y, Ohno M, et al. Effects of chronic vardenafil treatment persist after end of treatment in rats with acute arteriogenic erectile dysfunction. J Sex Med 2012;9:1782-8.
6. Kwon MH, Tuvshintur B, Kim WJ, Jin HR, Yin GN, Song $\mathrm{KM}$, et al. Expression of the apelin-APJ pathway and effects on erectile function in a mouse model of vasculogenic erectile dysfunction. J Sex Med 2013;10:2928-41.

7. Park K, Son H, Kim SW, Paick JS. Initial validation of a novel rat model of vasculogenic erectile dysfunction with generalized atherosclerosis. Int J Impot Res 2005;17:424-30.

8. Ryu JK, Cho CH, Shin HY, Song SU, Oh SM, Lee M, et al. Combined angiopoietin-1 and vascular endothelial growth factor gene transfer restores cavernous angiogenesis and erectile function in a rat model of hypercholesterolemia. Mol Ther 2006;13:705-15.

9. Ryu JK, Shin HY, Song SU, Oh SM, Piao S, Han JY, et al. Downregulation of angiogenic factors and their downstream target molecules affects the deterioration of erectile function in a rat model of hypercholesterolemia. Urology 2006;67: 1329-34.

10. Shiota A, Hotta Y, Kataoka T, Morita M, Maeda Y, Kimura K. Oral L-citrulline supplementation improves erectile function in rats with acute arteriogenic erectile dysfunction. J Sex Med 2013;10:2423-9.

11. Akaihata H, Nomiya M, Hata J, Yabe M, Takahashi N, Haga N, et al. Pelvic arterial occlusive disease affects the RhoA/Rhokinase pathway in bladder smooth muscle. J Urol 2015;193: 706-13.

12. Goi Y, Tomiyama Y, Nomiya M, Sagawa K, Aikawa K, Yamaguchi O. Effects of silodosin, a selective a1A-adrenoceptor antagonist, on bladder blood flow and bladder function in a rat model of atherosclerosis induced chronic bladder ischemia without bladder outlet obstruction. J Urol 2013;190:1116-22.

13. Nomiya M, Andersson KE, Yamaguchi O. Chronic bladder ischemia and oxidative stress: new pharmacotherapeutic targets for lower urinary tract symptoms. Int J Urol 2015;22:406.

14. Nomiya M, Yamaguchi O, Andersson KE, Sagawa K, Aikawa $\mathrm{K}$, Shishido K, et al. The effect of atherosclerosis-induced chronic bladder ischemia on bladder function in the rat. Neurourol Urodyn 2012;31:195-200.

15. Shim JS, Kim DH, Bae JH, Moon du G. Effects of omega-3 fatty acids on erectile dysfunction in a rat model of atherosclerosis-induced chronic pelvic ischemia. J Korean Med Sci 2016;31:585-9.

16. Kim JH, Lee HJ, Song YS. Mesenchymal stem cell-based gene therapy for erectile dysfunction. Int J Impot Res 2016;28:81-7.

17. Pelliccione F, D’Angeli A, Filipponi S, Falone S, Necozione S, Barbonetti A, et al. Serum from patients with erectile dysfunction inhibits circulating angiogenic cells from healthy men: relationship with cardiovascular risk, endothelial dam- 
age and circulating angiogenic modulators. Int J Androl 2012; 35:645-52.

18. Williams JK, Andersson KE, Christ G. Animal models of erectile dysfunction (ED): potential utility of non-human primates as a model of atherosclerosis-induced vascular ED. Int J Impot Res 2012;24:91-100. 